\title{
Ivermectin in Long-Covid Patients: A Retrospective Study
}

\author{
Del Franco Haroldo ${ }^{*}$, Carvallo Hector ${ }^{2}$, Hirsch Roberto ${ }^{3}$
}

\author{
${ }^{1}$ Neumologist, Mercante Public Hospital, Argentina \\ ${ }^{2}$ Professor of Internal Medicine, U.A.I, Argentina \\ ${ }^{3}$ Professor of Professor of Infectology, U.B.A. Buenos Aires, Argentina
}

\begin{abstract}
Long COVID convalescence has become a major issue in COVID infection. The variety and magnitude of sequelae has, so far, baffled scientific community, and no measure has proved to be both useful and reliable in diminishing and/or shortening it. We are summarising the outcomes in 856 patients previously admitted at a Public Hospital in the Province of Buenos Aires, due to moderate/severe COVID infection, who surmounted the infection and could be released later on.

We selected those whose symptoms, and mainly, the duration of them- could be attributed to long convalescence (long haulers).

In them, a simple post-COVID treatment with ivermectin (IVM) was applied, thus provoking a faster reduction of manifestations.
\end{abstract}

Keywords: long COVID, Ivermectin, long haulers.

\section{Introduction}

\section{Current Knowledge on Long Covid:}

There has been an increasing number of reports of COVID-19 symptoms extending beyond the acute phase of infection, named "long COVID"; and its sufferers are known as "long haulers". A range of multiorgan complications following COVID-19 infection - including respiratory, cardiovascular, metabolic and renal impairments - have also been hypothesised [1].

In a survey by the UK Government's Office for National Statistics in November, 2020, around one in five people who tested positive for COVID-19 had symptoms that lasted for 5 weeks or longer, and one in ten people had symptoms that lasted for 12 weeks or longer. These figures equate to an estimated 186000 individuals in England who had symptoms persisting between 5 and 12 weeks [2].

Adults with severe illness who spend weeks in intensive care, often intubated, can experience long-lasting symptoms, but that's not unique to patients with COVID-19.

What's unusual about the long haulers is that many initially had mild to moderate symptoms that didn't require lengthy hospitalization-if any let alone intensive care.
In some organs, especially the lungs, those changes persist far past the point at which patients have stopped shedding the virus [3].

The list of long hauler symptoms is long, wide and inconsistent. The most common long hauler symptoms include (Fig-1):

- Coughing

- Ongoing, sometimes debilitating, fatigue

- Body aches

- Joint pain

- Shortness of breath

- Loss of taste and smell-even if this didn't occur during the height of illness

- Difficulty sleeping

- Headaches

- Brain fog

Corresponding Author: Del Franco Haroldo, Neumologist, Mercante Public Hospital, Argentina Received date: March 09, 2021; Accepted date: March 17, 2021; Published date: March 18, 2021.

DOI: https://doi.org/10.31546/2633-8653.1008 


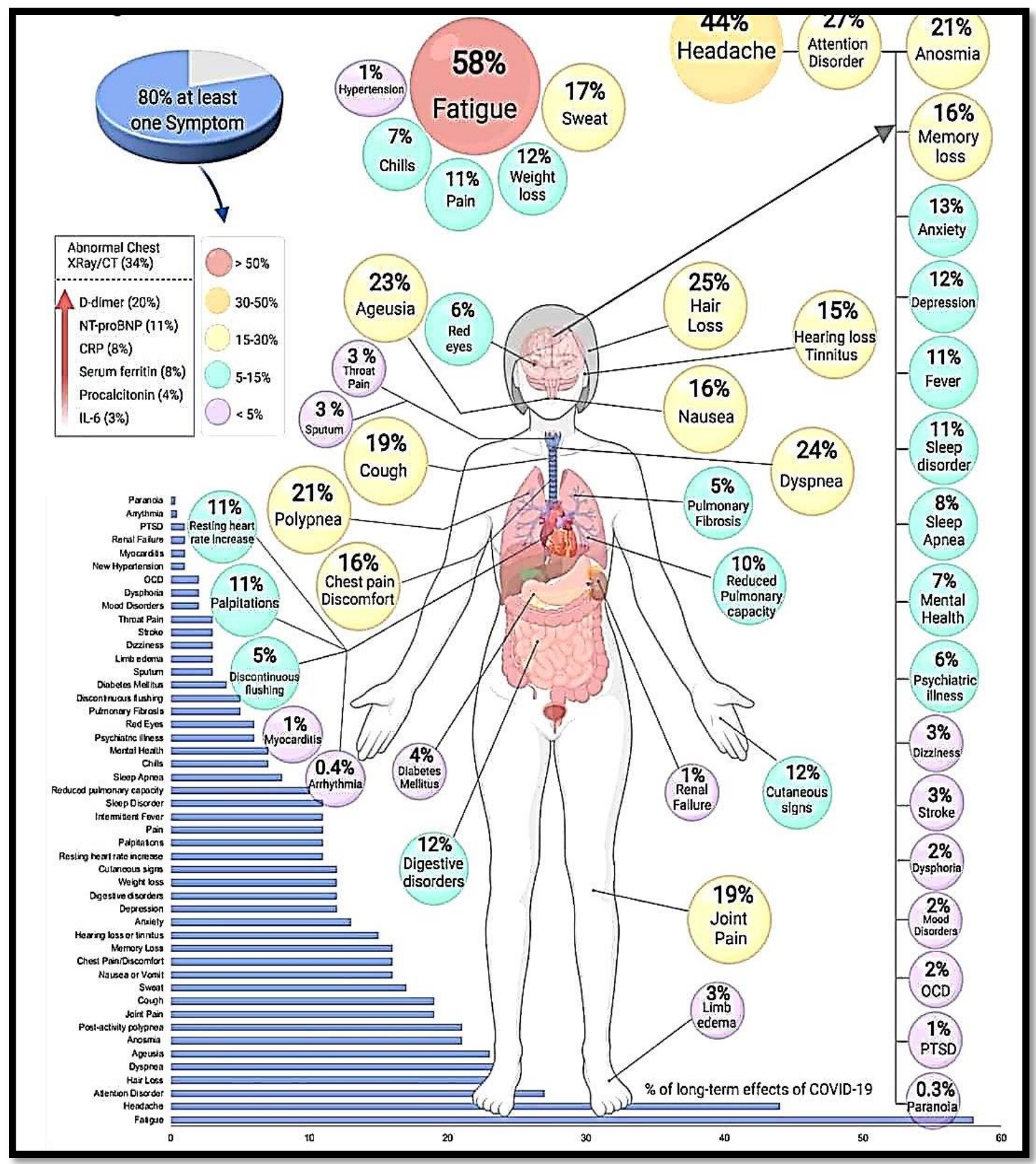

Figure 1: Long-Term Covid Effects (From Sonia Villapol: Long Term Effects of Covid 19. The Conversation. February, 2021)

Brain fog is among the most confusing symptoms for long haulers. Patients report being unusually forgetful, confused or unable to concentrate.

This can happen to people who were in an intensive care unit for a while, but it's relatively rare.

Any well-trained Medical Doctor can tell -from the way a COVID-patient talks- if he is still suffering a brain fog, as the voice sounds like that of a nearly drunken person.
There's not a lot of information on long haulers, who only recently received attention from experts because it's also so new.

The vast majority of long haulers test negative for COVID-19. One theory about patients with long-term COVID-19 symptoms is that the virus possibly remains in their bodies in some small form. 
Another theory is their immune systems continue to overreact even though the infection has passed [4].

Based on the experience with other viral epidemics that are capable of causing a chronic-phase of the disease, COVID-19 is difficult because of the diversity it exhibits in:

a- Evading and affecting the human immune system.

b- The tissue tropism it exhibits based on ACE2 receptor density. c- Its capability to run amok with multiple organ and systems.

Factors like the viral load, which may get eradicated or persist resulting from tissue budding of SARS-CoV-2, appear to be possibly playing the main role in longhaulers (Fig-2).

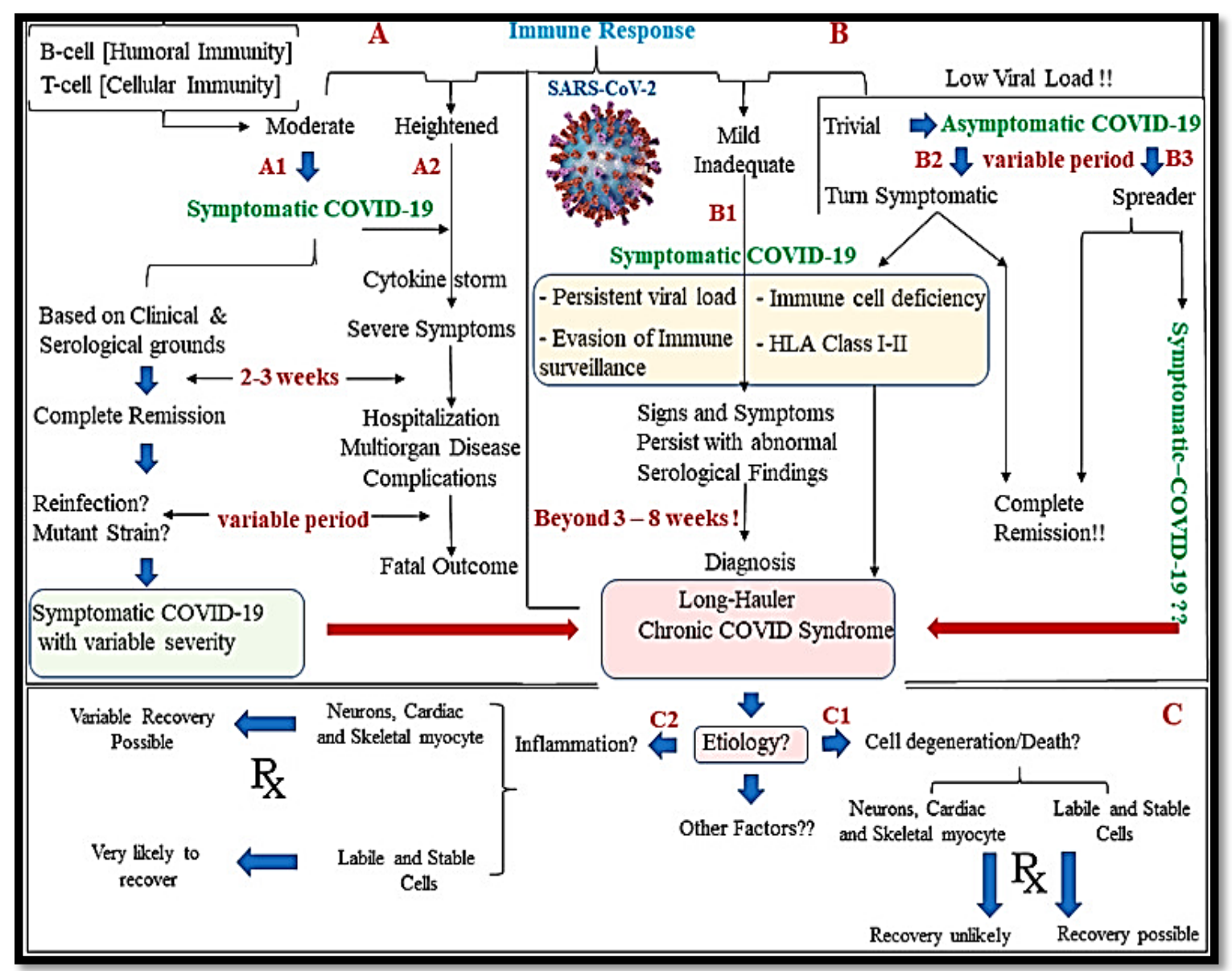

Figure 2: Phisiopathogenic Pathways towards long Covid (from: Abdul Mannan Baig: Deleterious Outcomes in Long-Hauler COVID-19)

Other factors like ACE2 density in tissues, vascular permeability, coagulation, and cytokine activation cascade appear to determine a progression to a prolonged, less lethal but more incapacitating clinical picture [5].

This could lead to a significant surge of people battling lasting illnesses and disabilities. Symptoms lasting several weeks and impairing a person's usual function should not be called mild, neither should the subject be considered up to returning to usual activities so quickly. Restoring such a subject to his ordinary labour tasks is a potential risk for both himself and his fellow co-workers.
Defining and measuring recovery from COVID-19 should be more sophisticated than checking for hospital discharge, or testing negative for active infection or positive for antibodies. Once recovery is defined, we can differentiate COVID that quickly goes away from the prolonged form [6].

The terms "COVID long haulers," "long COVID," and "Post COVID Syndrome," have all been used interchangeably in recent months to describe individuals who have been infected with the SARSCoV-2 and continue to experience symptoms after "recovery." 
However, these terms may be misleading, as they represent three different patient subsets with prolonged symptoms, usually defined as greater than one month after COVID-19 infection.

\section{These subsets include:}

a. Critically-ill patients, almost all who have been admitted to intensive care, who, are expected to have a lengthy recovery period (often months) and may have permanent organ damage, particularly in the lung.

b. An undetermined number of patients, following mild or severe infection, who have organ damage/dysfunction, such as myocarditis or an encephalopathy. The long-range consequences are unclear.

c. Many patients, in some series estimated up to $10 \%$, have prolonged, multisystem symptoms with no evidence of organ damage or dysfunction.

These patients most often have severe exhaustion, headaches, myalgias, and mood and cognitive disturbances with normal physical and laboratory findings. This is the subset experiencing symptoms most similar to post-viral fatigue syndrome (PVFS), chronic fatigue syndrome (CFS) - also termed benign myalgic encephalomyelitis (BME) in the UK, fibromyalgia and other related, poorly understood disorders associated with chronic fatigue and pain. In these conditions, there has been no strong evidence for organ damage or persistent and significant immune/inflammatory abnormalities.

The confusion and controversy playing out online and among support groups for the third subset of patients mirrors the misunderstandings of CFS over the past 40 years in both the United States and the United Kingdom.

The symptoms are similar to those reported in PVFS or CFS. However, dyspnea and loss of taste and smell appear to be much more common in Post COVID Syndrome patients.

While PVFS is not new to the medical community, there is no clear path to treatment. More important, there is still not enough knowledge about the exact causes or repercussions of post-viral fatigue in recovering COVID-19 patients.

Ongoing symptoms reported by those recovering from COVID-19 infection should be taken seriously and addressed accordingly. Beyond doubt, there are a considerable number of individuals who have a post-viral syndrome that really, in many respects, can incapacitate them for weeks and weeks following so-called recovery and clearing of the virus, highly suggestive of myalgic encephalomyelitis/chronic fatigue syndrome [7].
A new study published in the Annals of the American Thoracic Society, experts concede they don't know why some people who develop COVID-19 experience persistent symptoms after the virus is no longer detectable in their body. When healthcare providers order chest X-rays, CT scans, or other tests to look for potential causes of long-haul symptoms, the results often come back negative.

Over 60 percent of the study participants said they had not yet returned to full health an average of 75 days after their diagnosis. However, only 4 percent showed signs of lung scarring on CT scans [8]. Those who emerged from life support at ICU, or beat severe cases of COVID-19 in the hospital, are being home- visited and/or receive virtual house calls tend to post-hospital diagnoses, medical checks as well as physical and occupational therapy for patients dealing with symptoms impacting cognitive function as well as anxiety or depression [9].

Outpatient clinics that are dedicated to following up on lasting disabilities in the large number of patients who previously had COVID-19 are opening in many hospitals, especially in areas where large SARS-CoV2 outbreaks have occurred. However, this initiative implies a further burden on the health-care system in terms of human and economic resources, in addition to conventional health-care services. Unfortunately, these clinics are largely unaffordable in most low-income or middle-income countries that have also been severely affected by the COVID-19 pandemic. This is consistent with the syndemic nature of the COVID-19 pandemic, and has implications for the long-term follow-up of COVID-19 sequelae, which in most instances should be interpreted against a background of an array of non-communicable diseases and social and income inequalities that exacerbate the adverse effects of each of these diseases in many communities [10].

On the other hand, trying to make these patients recover by just prescribing "vitamins, deep breath and plenty of rest" brings us back to the XIX century methods [11].

J. George characterized a group consisting of mostly outpatients: $90 \%$ of their cohort experienced only a mild COVID-19 illness, yet one-third continue to have lingering effects. Many of those individuals were young and have no pre-existing medical conditions, 
indicating that even relatively healthy individuals may face long-term impacts from their illness.

Early in 2021, Wuhan studies of non-hospitalized patients have shown that anywhere from $35 \%$ to $50 \%$ of non-hospitalized patients had symptoms 2 to 4 months later.

Fatigue, breathing issues, and cardiac concerns like chest pain are common findings, as are neurologic symptoms. Of patients at the Center for Post-COVID Care at Mount Sinai with neurology referrals, about $65 \%$ come in with cognitive complaints or brain fog. Brain fog means different things to different people, but usually it's some combination of shortterm memory issues, attention issues, and word-finding difficulty.

The University of Washington study followed 177 people with laboratory-confirmed SARS-CoV-2 infection who completed questionnaires from August to November 2020, 3 to 9 months after their COVID-19 onset (median 169 days).

Mean age was 48 and 57\% were women. Hypertension was the most common comorbidity (13\%).

Across the cohort, $6.2 \%$ of participants were asymptomatic, $84.7 \%$ were outpatients with mild illness, and $9.0 \%$ were hospitalized with moderate or severe disease. Patients completed followup questionnaires a median of 169 days after COVID-19 onset.

Overall, $32.7 \%$ of outpatients and $31.3 \%$ of inpatients reported at least one persistent symptom, most commonly fatigue (13.6\%) and loss of sense of smell or taste (13.6\%). In addition, $13.0 \%$ reported other symptoms, including brain fog $(2.3 \%)$.

Among outpatients and hospitalized patients, 30.7\% reported worse health-related quality of life compared with baseline; this figure was $12.5 \%$ for patients who never had overt COVID symptoms.

About $8 \%$ of all participants said at least one activity of daily living suffered long-term consequences, most commonly household chores.

Study limitations include small sample size, single study location, and potential bias from self-reported symptoms [12].

a. Putting all the above-mentioned facts and theories in perspective, some obvious certainties arise: The real number of long-COVID patients is not clear.

b. There is no close relation between the severity of the illness and the long COVID. c. The origin of this syndrome may be related to the permanence of the virus in tissues either tan blood.

d. There is no current treatment for reducing long COVID.

\section{Current Knowledge about Ivermectin (IVM)}

Ivermectin (IVM) is an antiparasitic (endodecticide), with nematicidal and ectoparasiticidal properties. It is a macrocyclic lactone derived from avermectins, a group of highly active endodecticidal antiparasitic agents isolated by fermentation of the soil microorganism Streptomyces avermitilis.

It was discovered in 1960 in Japan by Satoshi Omura. In 1981, William C. Cambell began the studies that allowed its veterinary use.

Both received the Nobel Prize in Physiology and Medicine in 2015.

In 1985, the French proved its usefulness in onchocerciasis in Africa. It was approved in 1997 by the FDA for treatments of: strongylidiasis and crusted scabies, in patients with AIDS.

In Human Medicine, it has been used in children from 5 years of age onwards, for the management of ecto and enteroparasitosis.

Orally, in humans, it does not cross the blood-brain barrier. It is contraindicated in pregnancy and lactation.

Recently, its viricidal effects on flaviviruses, dengue, Zica, Chikungunya, among others, have been compiled.

Ivermectin is an inhibitor of the causative virus (SARS-CoV-2).

This activity is due to the dependence of many RNA viruses on IMP $\alpha$ / $\beta 1$ during infection. Recently, another mechanism of action has been proposed, assuming its role as an ionophore agent.

Ionophores have many oxygen atoms internally, and are essential for binding cations and transporting them through phospholipid bilayers (cell membranes; phospholipid capsid of the virus). As a consequence, it determines an ionic imbalance between the external and internal environment, with the consequent osmotic 
These results, as a whole, demonstrated that ivermectin possesses antiviral action against SARS-CoV-2 in vitro, with a single dose capable of controlling viral replication in 24-48 hours, and the possibility of repeating it periodically.

Research has been added on other forms of action of IVM in the face of COVID disease, not directly on the causative agent, but on the pathophysiological mechanisms through which its deleterious activity develops (hyperinflammation and hypercoagulability). All of the above is summarized in Figure 3.

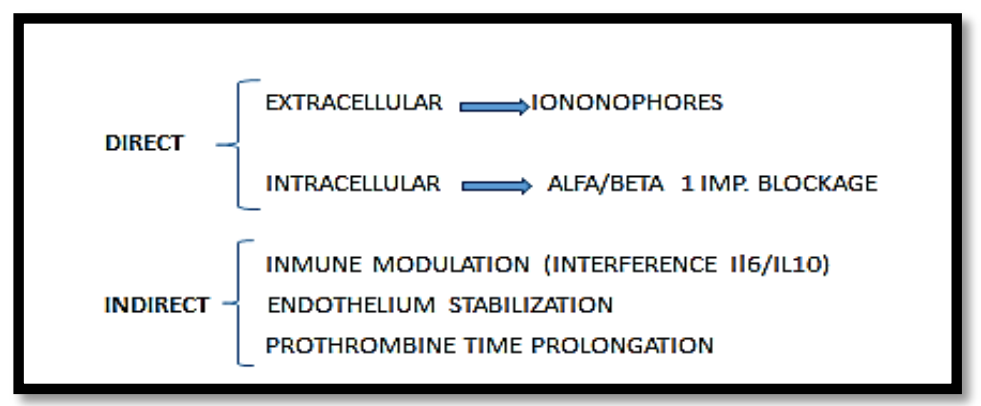

Figure 3: IVM Mechanisms of Action (from Hirsch Roberto R, Carvallo Hector E.: COVID 19 and Ivermectin Prevention and Treatment Update)

The effectiveness of IVM on pre and intra COVID have already been proved in all our essays $[13,14,15]$. Though still neglected by the W.H.O., from the statistical point of view, IVM has already proved its effectiveness against COVID 19 infection in all stages of the disease. A meta-analysis also proved that an early treatment is far more effective than a delayed one. The amount of data supporting the need to incorporate IVM amid the options of treatment against COVID 19 is countless, and -little by Little- more Countries and regions around the World are including it in their schemes (Fig-4).

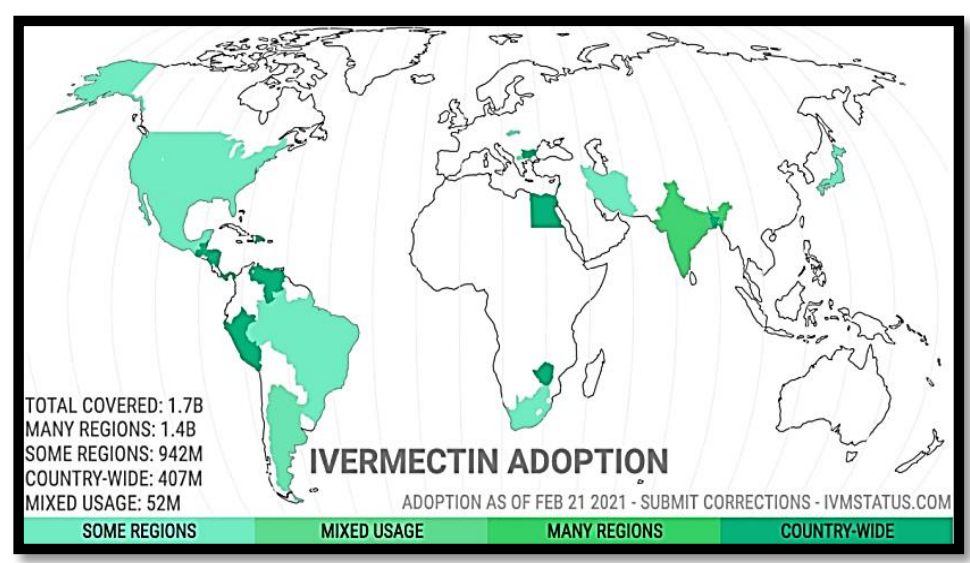

Figure 4: IVM use against COVID 19 in the World, February 2021 (from IVM Meta)
Our figures account for a $95.5 \%$ reduction in the need of hospitalization, and a 95,6\% reduction in death rate, when IVM treatment was applied at the early stages of the disease, in consonance with existing data. According to ours and other international data, roughly 11,000 lives worldwide could have been saved daily, by simply adding IVM as the treatment offered to COVID 19 patients [16].

Statistically speaking, up to $10 \%$ of mild cases, and up to $40 \%$ of moderate cases, require hospitalization. For this reason, avoiding this instance is crucial, not only to decompress health systems, but also to drastically lower their costs, which will result in the possibility of sustaining these health policies indefinitely. We also carried out a cost-survey to support what has been stated so far: Hospitalization Costs, in Europe: Daily hospitalization (average): 2,932 euros this figure doubles, if the ICU admission is considered. IVM presentation of $20 \mathrm{ml}$ (for two weeks): 5 euros [17].

\section{Material and Method:}

We conducted a follow-up of 856 patients discharged from hospitalization at a Public Hospital in Buenos Aires (Argentina), starting on July 2020 and still continuing. All the patients included had not received IVM either before or during hospitalization. Instead, they had been treated with corticosteroids, antibiotics, blood-thinners, convalescent's plasma, etc.

The follow-up lasted as long as the patients were found to be free from symptoms and/or sequelae. Thus, some of them remain in weekly observation. For that reason, we divide this cohort into two groups:

a- Those patients who could finally be discharged even from ambulatory control (799).

b- Those patients who still have to be controlled, or referred to different subspecialties (57).

Beginning by this Second Group, the long-lasting symptoms have been:

1) Stress and/or paranoid disorders (39 from a total of 57).

2) Memory loss (8 from a total of 57).

3) Dysgeusia, defined as alterated sense of taste, mainly mixing salad and sweet sensations (6 from a total of 57).

4) Fatigue (4 from a total of 57).

All these 57 patients had been at ICU for over 10 days. 
Now turning to the FIRST GROUP (799 subjects) it was composed of 411 male $(51.44 \%)$ and 388 female individuals $(48.56 \%)$. (Figure 5)

The average age was 52,3 years, ranging from 21 to 82 years. (Figure 6)

a. The prevailing symptoms were:

b. Coughing (not related to previous history of COLD)

c. Brain fog (not related to previous circulatory diseases and/or dementia)

d. Headaches (not related to high blood pressure or other common causes)

e. Persistent fatigue (not related to anemia and/or other causes)

f. Loss of taste and smell

g. Shortness of breath (same as "a" group)

h. Body/joint aches (not related to previous history of arthritis) (Figure 7)

Some patients had two or more simultaneous symptoms.

All of the above-mentioned patients received IVM, ranging from 12 up to $18 \mathrm{mg}$ per os, on a weekly basis, until the symptoms disappeared, but no longer than 8 weeks (what happened first).

Some of them also received polivitaminic compounds (the exact number lacking, because it was an auto-prescription, but not exceeding from 12 patients).

The average time needed to get rid of those disturbing symptoms were 36 days, ranging from 21 to 69 days. (Figure 8 and 9)

The side effects reported by the IVM-treated patients were:

1) Diarrheic episodes in 5 patients

2) Abdominal pain in 2 patients

No cases of allergy were reported; neither were pregnant or lactating women among those treated, thus IVm contraindications could be disregarded.

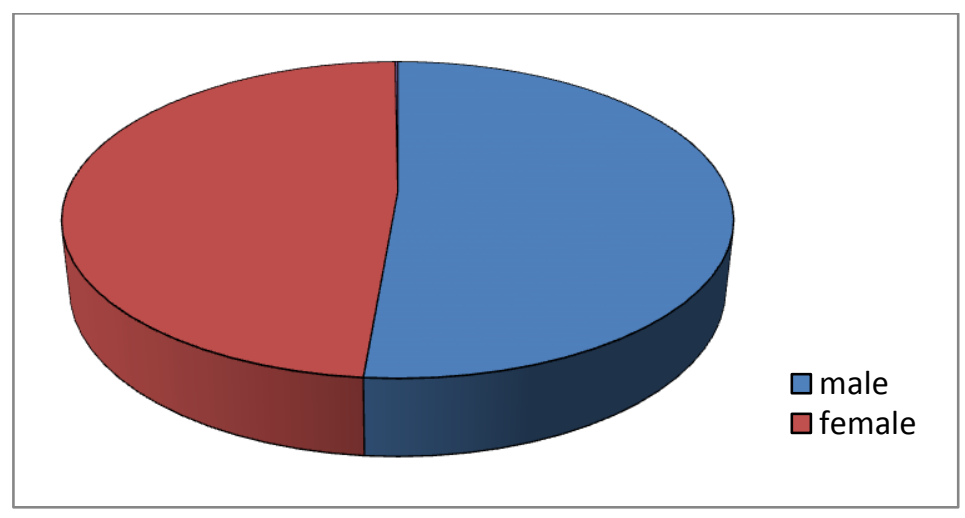

Figure 5: Sex-Related Distribution

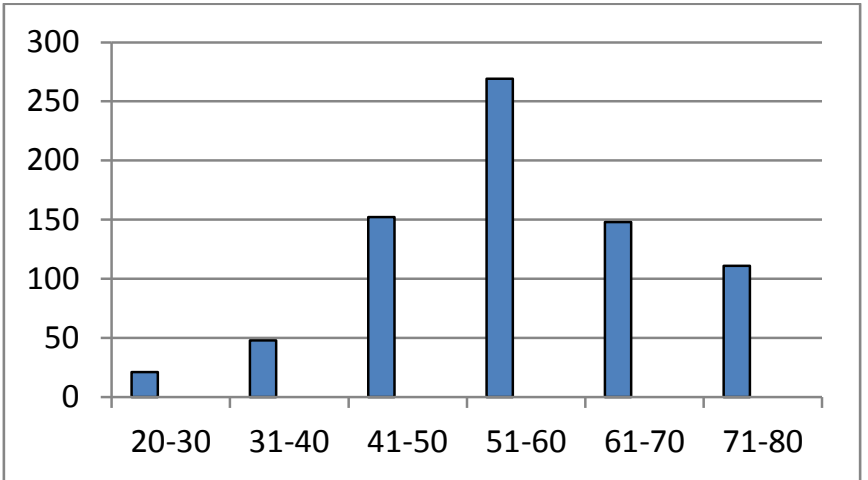

Figure 6: Age-Related Distribution

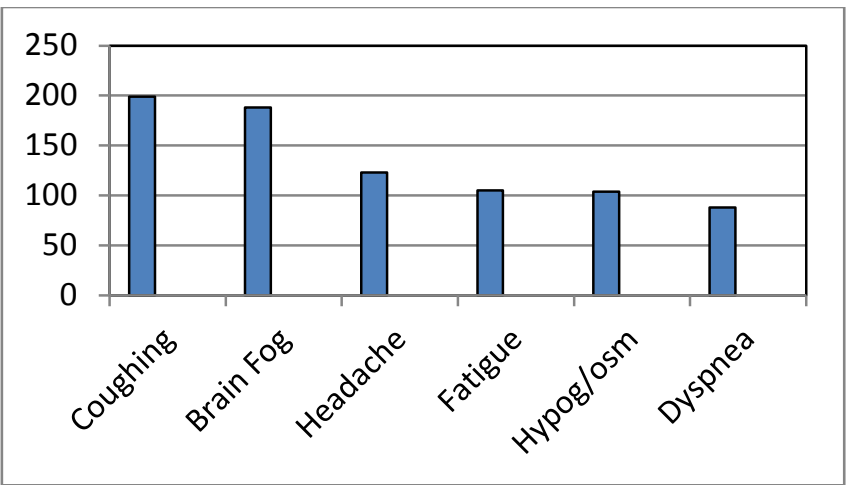

Figure 7: Symptoms Referred by Post-COVID Patients

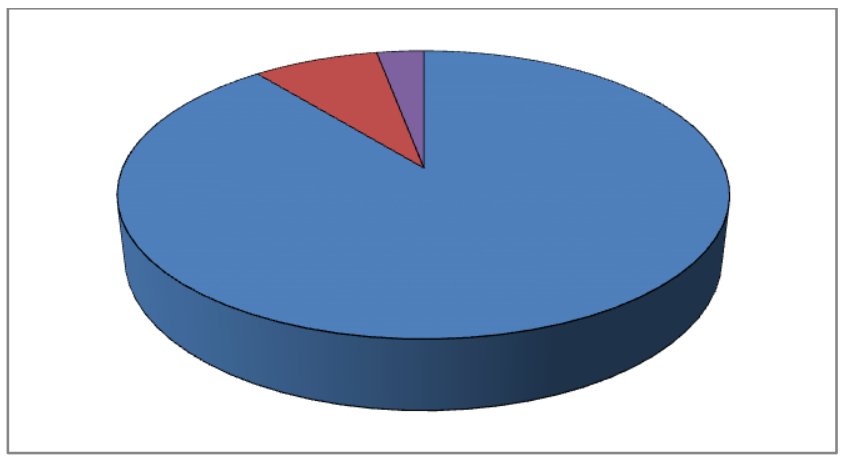

Figure 8: Days to make symptoms disappear in IVM-Treated and Untreated Patients (in red and violet: patients on IVM; in blue: collected data from different surveys of untreated patients)

\section{Conclusion}

Long COVID includes a constellation of symptoms, that may be caused by the unobtrusive persistence of virus in different tissues, and the subsequent persistence of inflammatory and coagulation disorders. Since IVM has already proved to impair virus capacity to invade cells, and also to have immune and clotting modulatory effects, there is a reasonable chance to diminish, shorten and even completely correct almost all the symptoms by using IVM in the post COVID.

Consent for Publication: Written informed consent was obtained from the patient for the publication. 
Conflicts of Interest: The authors declare no conflict of interest.

\section{References:}

1. Office for National Statistics (ONS), UK; The prevalence of long COVID symptoms and COVID-19 complications. Last updated: 16 December 2020.

2. Priya Venkatesan. NICE guideline on long COVID. Lancet Respir Med. 2021;9:129.

3. Rita Rubin. As Their Numbers Grow, COVID-19 "Long Haulers" Stump Experts. JAMA.2020;324 :1381-1383.

4. UC Davis Health: Long haulers: Why some people experience long-term coronavirus symptoms. Updated Feb. 8, 2021.

5. Abdul Mannan Baig. Deleterious Outcomes in LongHauler COVID-19: The Effects of SARS-CoV-2 on the CNS in Chronic COVID Syndrome. ACS Chem Neurosci. 2020;11:4017-4020.

6. Nisreen AA. A negative COVID-19 test does not mean recovery. Nature. 2020;584:170.

7. Jennifer L. COVID Long Haulers: Symptoms and Connections to Post Viral Fatigue and Organ Damage. Practical Pain Management. December 22, 2020.

8. Liam Townsend, Joanne D, Kate O'Brien, et al. Persistent Poor Health Post-COVID-19 Is Not Associated with Respiratory Complications or Initial Disease Severity. Ann Am Thorac Soc. January 8, 2021.

9. Jim A. 'Long Haulers': URMC Treating Lingering COVID Symptoms in Coronavirus Survivors. Spectrum Helth News, Rochester. January 07, 2021.
10. Monica C, Norberto P, Giuseppe R. Long-term follow-up of recovered patients with COVID-19. Lancet. 2021;397:173-175.

11. Aaron BH. Treating Post-COVID Fatigue and Dyspnea; Deep Breaths and Plenty of Rest. Medscape. Febuary 19, 2021.

12. Judy George. Study Puts Numbers to 'Long COVID' Duration, Prevalence. MedPage Today. February 19, 2021.

13. Carvallo H, Hirsch R, Farinella ME. Safety and efficacy of the combined use of ivermectin, dexamethasone, enoxaparin and aspirin against covid 19. MedRxiv preprint. September 15, 2020.

14. Carvallo Héctor, Hirsch Roberto, Alkis Psaltis, Contreras Veronica: Study of the Efficacy and Safety of Topical Ivermectin + IotaCarrageenan in the Prophylaxis against COVID-19 in Health Personnel. J Biomed Res Clin Investig. 2020;2:1007.

15. Hirsch Roberto R, Carvallo Hector E. Covid 19 and Ivermectin Prevention and Treatment Update. Inf Dis Trav Med. 2020;4:1-4.

16. Alonso L, Bracho C, Wilmer C, et al. Ivermectin in Covid-19 patients: a multicenter, retrospective study. Researchgate. January 2021.

17. Carvallo HE, Matozza F, Hirsch RR. Covid Pandemic and Repurposed Drugs: How much is a human life? Researchgate. Febuary 2021. 